\title{
COVID-19-Related Intracerebral Hemorrhage
}

\author{
Valentin Pavlov ${ }^{1,2}$, Ozal Beylerli ${ }^{1}$, Ilgiz Gareev ${ }^{1}$, Luis Fernando Torres Solis ${ }^{3}$, \\ Arturo Solís Herrera ${ }^{4}$ and Gjumrakch Aliev ${ }^{5,6,7,8 *}$
}

\begin{abstract}
${ }^{1}$ Central Research Laboratory, Bashkir State Medical University, Ufa, Russia, ${ }^{2}$ Department of Urology, Bashkir State Medical University, Ufa, Russia, ${ }^{3}$ The School of Medicine, Universidad Autónoma de Aguascalientes, Aguascalientes, Mexico, ${ }^{4}$ Human Photosynthesis ${ }^{\odot}$ Research Centre, Aguascalientes, Mexico, ${ }^{5}$ Sechenov First Moscow State Medical University, Sechenov University, Moscow, Russia, ${ }^{6}$ Research Institute of Human Morphology, Russian Academy of Medical Sciences, Moscow, Russia, ${ }^{7}$ Institute of Physiologically Active Compounds, Russian Academy of Sciences, Chernogolovka, Russia, ${ }^{8}$ GALLY International Biomedical Research Institute, San Antonio, TX, United States
\end{abstract}

OPEN ACCESS

Edited by:

Thomas Wisniewski,

New York University, United States

Reviewed by:

Archana Hinduja,

The Ohio State University,

United States

Lauren Allegra Beslow,

The Children's Hospital of

Philade/phia, United States

*Correspondence:

Gjumrakch Aliev

aliev03@gmail.com;

cobalt55@gallyinternational.com

Received: 29 August 2020 Accepted: 28 September 2020 Published: 22 October 2020

Citation:

Pavlov V, Beylerli O, Gareev I, Torres Solis LF, Solís Herrera A and Aliev G (2020) COVID-19-Related

Intracerebral Hemorrhage.

Front. Aging Neurosci. 12:600172. doi: 10.3389/fnagi.2020.600172
Intracerebral hemorrhage $(\mathrm{ICH})$ is a common and severe neurological disorder and is associated with high rates of mortality and morbidity. $\mathrm{ICH}$ is associated with old age and underlying conditions such as hypertension and diabetes mellitus. The COVID19 pandemic is associated with neurological symptoms and complications including $\mathrm{ICH}$. For instance, the mechanisms by which COVID-19 may contribute to hemorrhagic stroke may include both depletion of angiotensin converting enzyme 2 (ACE2) receptor and overactive immune response. In this study, we herein report three patients (0.25\%) out of 1200 admissions with COVID-19 to our center between 1 May and August 4, 2020, who developed ICH. In addition, we will briefly discuss the possible pathophysiological mechanisms of COVID-19 infection in patients with $\mathrm{ICH}$.

Keywords: COVID-19, intracerebral hemorrhage, pathophysiological mechanisms, neurological consequences, complications

\section{INTRODUCTION}

Coronavirus infection 2019 (COVID-19) is a dangerous infectious disease that occurs as an acute respiratory viral infection with specific complications, which may include pneumonia, which leads to acute respiratory distress syndrome or respiratory failure with a high risk of death. Among the main complications of COVID-19 on the nervous system are encephalopathy, encephalitis, acute disseminated encephalomyelitis, meningitis, ischemic stroke, and intracerebral hemorrhage (ICH) and other diseases (Kannan et al., 2020). A growing number of case reports and series have been published describing the clinical characteristics of patients with ICH and COVID-19 (Table 1). The clinical course of COVID-19 is most severe in the elderly, and in patients with concomitant diseases such as hypertension and diabetes mellitus (DM), which are known to be the main factors in the development of ICH (Divani et al., 2020). Stroke has been reported as a complication of COVID-19. Most strokes among COVID-19 patients are arterial ischemic, though ICH has also been reported. We present three cases of ICH among patients hospitalized with COVID-19 from 1 May to August 4, 2020. ICH represented an infrequent complication among patients hospitalized with COVID-19 at our center, and patients with COVID-19 and ICH had other risk factors for ICH. However, our patients along with others in the literature suggest that several mechanisms may contribute to ICH in the setting of COVID-19. 
TABLE 1 | Summary of published cases of COVID-19-related ICH.

\begin{tabular}{|c|c|c|c|c|}
\hline Study & Total COVID-19, n & Patients with $\mathrm{ICH}, \mathrm{n}$ & Vascular risk factors & Mortality rate, $\%$ \\
\hline Reddy et al., 2020 & 47 & 1 & Hypertension & $0 \%$ \\
\hline Li et al., 2020 & 221 & 1 & Not reported & $100 \%$ \\
\hline Morassi et al., 2020 & 6 & 2 & Hypertension and thrombocytosis & $100 \%$ \\
\hline Al-olama et al., 2020 & 1 & 1 & Meningoencephalitis & $100 \%$ \\
\hline Bao et al., 2020 & 1 & 1 & None & $0 \%$ \\
\hline Benger et al., 2020 & 5 & 5 & Hypertension, IHD, T2DM, and anticoagulant therapy & $0 \%$ \\
\hline Sharifi-Razavi et al., 2020 & 1 & 1 & Not reported & Not Reported \\
\hline Haddadi and Shafizad, 2020 & 1 & 1 & Hypertension and diabetes & $0 \%$ \\
\hline Khattar et al., 2020 & 1 & 1 & Hypertension & $100 \%$ \\
\hline Kvernland et al., 2020 & 4071 & 16 & Hypertension, and anticoagulant therapy & $63 \%$ \\
\hline Daci et al., 2020 & 1 & 1 & None & $100 \%$ \\
\hline Poyiadji et al., 2020 & 1 & 1 & Not reported & Not Reported \\
\hline Hernández-Fernández et al., 2020 & 1683 & 5 & Hypertension, dyslipidemia, and T2DM & $20 \%$ \\
\hline Shahjouei et al., 2020 & 26,175 (156 stroke patients) & 25 & Hypertension, IHD, and T2DM & Not Reported \\
\hline Ghani et al., 2020 & 3 & 3 & Hypertension, anticoagulant therapy and T2DM & $100 \%$ \\
\hline Al-Dalahmah et al., 2020 & 1 & 1 & Not Reported & $100 \%$ \\
\hline Gogia et al., 2020 & 1 & 1 & Hypertension and dyslipidemia & $100 \%$ \\
\hline Nawabi et al., 2020 & 18 & 6 & Hypertension and diabetes & Not Reported \\
\hline Kim et al., 2020 & 1 & 1 & Hypertension & $0 \%$ \\
\hline Dogra et al., 2020 & 755 & 33 & $\begin{array}{l}\text { Hypertension, dyslipidemia, T2DM, and anticoagulant } \\
\text { therapy }\end{array}$ & $42 \%$ \\
\hline
\end{tabular}

$1 \mathrm{CH}$, intracerebral hemorrhage; IHD, Ischemic heart disease; T2DM, Type 2 diabetes mellitus.

\section{MATERIALS AND METHODS}

\section{Ethics Approval and Consent to Participate}

The study protocol was approved by the Hospital COVID19 Clinics BSMU in Ufa, Russia. (Within the department of Neurosurgery), Clinic of the Bashkir State Medical University, Ufa, Republic of Bashkortostan, Russia. All research was performed in accordance with Bashkir State Medical University guidelines and regulations, and the respective authors declare a statement confirming that informed consent was obtained from all of the participants' parents and/or their legal guardians. In addition to the guidelines described above, the authors of these manuscripts dealing with human transplantation research attesting that no organs/tissues were procured from prisoners.

\section{Human and Animal Rights}

No animals were used for studies that are base of this research. All the humans used were in accordance with the ethical standards of the committee responsible for human experimentation (institutional and national), and with the Helsinki Declaration of 1975 , as revised in $2013^{1}$.

We performed a retrospective chart review of all hospitalized cases with confirmed COVID-19 infection and ICH between May 1 and August 4, 2020 seen at Hospital COVID-19 Clinics BSMU in Ufa, Russia. Diagnosis of ICH was confirmed on neuroimaging with computed tomography (CT) of the brain. COVID-19 infection nucleic acid tests were performed on

${ }^{1}$ http://ethics.iit.edu/ecodes/node/3931 nasopharyngeal swabs using quantitative real-time polymerase chain reaction (qRT-PCR). Patients were included in the case series if they had tested positive for COVID-19 prior to their ICH and had continuing clinical features related to COVID19. Inclusion criteria were defined as patients with acute ICH on CT neuroimaging and additional radiological assessment of the chest who were positive for COVID-19 and suffered from acute neurological symptoms during a hospital stay. Each of the scans had an electronic clinical and, if applicable, pathology report associated with it. Electronic reports were reviewed to extract clinical, laboratory, pathology, and demographic data. Patients were excluded if they had a secondary ICH from the hemorrhagic transformation of ischemic infarction, brain tumor, cerebral aneurysm, or vascular malformation. Baseline patient characteristics were retrieved from medical records, including symptom onset, Glasgow Coma Scale (GCS), and modified Rankin Scale (mRS) at last medical evaluation or at discharge. Additionally, vascular risk factors (hypertension, dyslipidemia and $\mathrm{DM}$ ), laboratory parameters (C-reactive protein, $\mathrm{D}$-dimer, etc.), and invasive procedures such as craniotomy from patients' clinical records and follow-up CT were obtained. Any missing or uncertain records were collected and clarified through direct communication with health care clinicians.

\section{RESULTS}

A total of 1200 patients with COVID-19 were hospitalized during the 65-day study; of these, we identified three patients $(0.25 \%)$ who presented with radiographic evidence of ICH and qRTPCR-confirmed COVID-19 infection. We describe their clinical 
characteristics, laboratory data, imaging findings, and clinical course (Table 2).

\section{CASE PRESENTATION}

\section{Patient 1}

A 50- year-old male with well-controlled hypertension presented with a 2-week history of cough, fever and fatigue. A CT chest study demonstrated bilateral ground-glass opacities consistent with COVID-19 pneumonia (Figure 1A). One week postadmission, he became drowsy with new severe headache, right-sided hemiplegia, and right-sided hemihypesthesia. A CT head examination demonstrated an ICH in the parietaloccipital region on the left with a breakthrough of blood into the ventricular system requiring craniotomy and evacuation (Figure 1D). An intracranial CT angiogram (CT-A) was normal. At the time of ICH detection, INR, APTT, platelets and fibrinogen were normal, and the patient was receiving a prophylactic dose of low molecular weight heparin (LMWH). After a 3-week admission, patient was discharged to a rehabilitation center for further therapy.

\section{Patient 2}

A 64-year-old male was hospitalized for COVID-19 pneumonia (7 days history of cough, fever and fatigue) treatment. A CT chest showed typical COVID-19 pneumonia changes (Figure 1B). He had a history of multiple, hypertension and type 2 diabetes mellitus. On day three of hospitalization, he developed left hemiparesis. He had a GCS score of 13 and a blood pressure of $210 / 90$. CT of the brain showed ICH in the projection of the basal ganglia on the right, which did not require surgical intervention (Figure 1E). CT-A of the brain was normal. The patient was admitted to the hyper-acute stroke unit (HASU) for further care. After a 2-week admission, he was discharged to a rehabilitation center for further therapy.

\section{Patients 3}

A 60 -year-old male with a history of hypertension, type 1 diabetes mellitus and hyperlipidemia, presented with a 5 days

TABLE 2 | Baseline characteristics of patients COVID-19 with new onset of ICH during infection.

\begin{tabular}{|c|c|c|c|}
\hline Characteristic & Patient 1 & Patient 2 & Patient 3 \\
\hline Age (years) & 56 & 64 & 60 \\
\hline Sex & Male & Male & Male \\
\hline Smoking history & Yes & Yes & No \\
\hline Blood pressure (mm Hg) (at the time of $\mathrm{ICH}$ detection) & $160 / 80$ & $210 / 90$ & $165 / 89$ \\
\hline Blood glucose (4.0-5.4 mmol/L) & 6.0 & 11.0 & 16.8 \\
\hline Red blood cells count $\left(4.5-5.7 \times 10^{12} / \mathrm{L}\right)$ & 3.8 & 3.0 & 2.8 \\
\hline Hemoglobin (115-160 g/L) & 96 & 68 & 75 \\
\hline Erythrocyte Sedimentation Rate (ESR) (<20 mm/h) & 18 & 27 & 21 \\
\hline White blood cells count $\left(4.0-11.0 \times 10^{9} / \mathrm{L}\right)$ & 14.8 & 15.0 & 15.2 \\
\hline Neutrophils $\left(1.8-7.5 \times 10^{9} / \mathrm{L}\right)$ & 12.5 & 12.3 & 14.8 \\
\hline Lymphocytes (1.5-4.0 × 109/L) & 1.8 & 1.6 & 3.8 \\
\hline Eosinophils $\left(0.0-0.4 \times 10^{9} / \mathrm{L}\right)$ & 0.0 & 0.0 & 0.1 \\
\hline Monocytes $\left(0.2-0.8 \times 10^{9} / \mathrm{L}\right)$ & 1.2 & 0.6 & 0.5 \\
\hline Basophils (0.0-0.15 × 109/L) & 0.0 & 0.1 & 0.0 \\
\hline Platelet count (150-450 × 109/L) & 220 & 334 & 480 \\
\hline Creatinine (45-120 mmol/L) & 66 & 138 & 130 \\
\hline Bilirubin, total (3-20 umol/L) & 18 & 12 & 37 \\
\hline Total cholesterol (<5.2 mmol/L) & 4.8 & 3.5 & 8.2 \\
\hline High-density lipoprotein (HDL) (>1 mmol/L) & 1.3 & 1.1 & 1.8 \\
\hline Low-density lipoprotein (LDL) (<3.4 mmol/L) & 2.8 & 3.5 & 5.17 \\
\hline D-Dimer (<500 ng/mL) & 1820 & 2580 & 4000 \\
\hline Fibrinogen (2.0 to $4.0 \mathrm{~g} / \mathrm{L})$ & 2.8 & 3.6 & 6.5 \\
\hline $\begin{array}{l}\text { Activated partial- thromboplastin time (aPTT) } \\
(20-35 \mathrm{~s})\end{array}$ & 22 & 25 & 21 \\
\hline International Normalized Ratio (INR) (0.85-1,15 ratio) & 1.0 & 1.1 & 1.1 \\
\hline C-reactive protein ( $<5 \mathrm{mg} / \mathrm{L})$ & 88 & 120 & 189 \\
\hline $\begin{array}{l}\text { Type of patients with COVID-19 } \\
\text { (severe/non-severe) }\end{array}$ & Severe & Severe & Severe \\
\hline Risk factors & Hypertension & $\begin{array}{l}\text { Type } 2 \text { diabetes, } \\
\text { hypertension }\end{array}$ & Hypertension, type 1 diabetes, high cholesterol, \\
\hline $\begin{array}{l}\text { The time between the onset of COVID-19 infection } \\
\text { and onset of } \mathrm{ICH} \text { (days) }\end{array}$ & 21 & 10 & 12 \\
\hline Location of hematoma & $\begin{array}{l}\text { Parietal-occipital region on the left } \\
\text { with a breakthrough of blood into } \\
\text { the ventricular system }\end{array}$ & $\begin{array}{l}\text { In the projection of } \\
\text { the basal ganglia on } \\
\text { the right }\end{array}$ & $\begin{array}{l}\text { In the projection of the basal ganglia on the right } \\
\text { with a breakthrough into the ventricular system } \\
\text { and a median dislocation to the left }\end{array}$ \\
\hline Modified Rankin Scale (mRS, 0-6) (discharge) & 4 & 2 & 5 \\
\hline
\end{tabular}



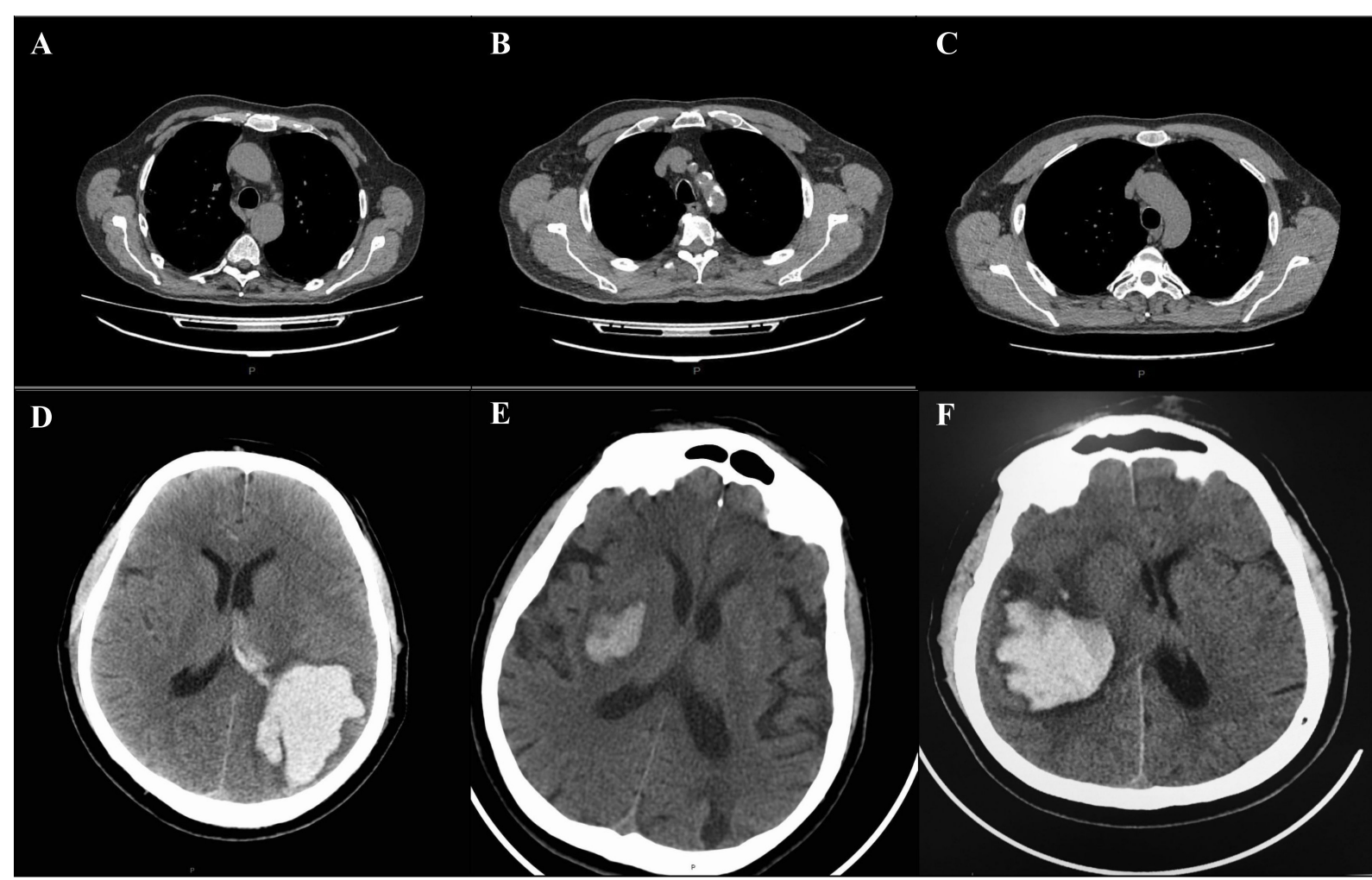

F
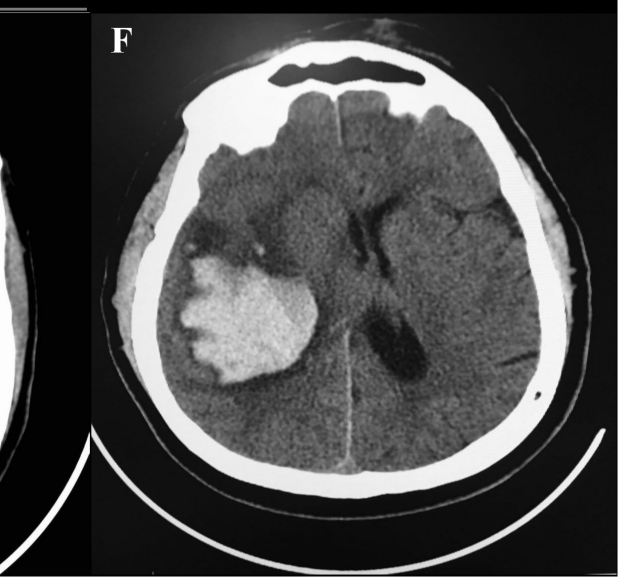

FIGURE 1 | CT changes of lung and brain in patients with COVID-19 complicated with ICH (A-F). Chest and brain CT examination of patient 1 showing bilateral consolidations and ground-glass opacities of the lungs (A), and hemorrhage in the parietal- occipital region on the left with a breakthrough of blood into the ventricular system (D). Chest and brain CT examination of patient 2, showing diffuse bilateral ground-glass opacities involving both lungs (B) and hemorrhage in the projection of the basal ganglia on the right (E). Chest and brain CT examination of patient 3, demonstrating bilateral ground-glass opacities (C) and hemorrhage in the projection of the basal ganglia on the right with a breakthrough into the ventricular system $\mathbf{( F )}$. $P=$ posterior.

history of shortness of breath, cough, fevers, and pleuritic chest pain. A CT chest study demonstrated bilateral ground-glass opacities consistent with COVID-19 pneumonia (Figure 1C). Clinical and laboratory evaluation showed moderate respiratory distress ( $\mathrm{PaO} 2 / \mathrm{FiO} 2$ 190). The respiratory function progressively worsened during the following days and, on day six, he was transferred to an intensive care unit (ICU) for invasive ventilation. The next day, the patient was found with bilaterally fixed and dilated pupils, with a GCS of 3. Brain CT examination showed a new ICH in the projection of the basal ganglia on the right with a breakthrough into the ventricular system requiring craniotomy and evacuation (Figure 1F). The ventricles were displaced across the midline. CT-A of the brain was normal. Coagulation tests were normal. A total of 6 weeks post-admission the patient remains on ICU, receiving multiple organ support.

\section{DISCUSSION}

We report three cases with severe COVID-19 infection who developed an ICH. Therefore, the question is whether COVID-19 infection is the cause of ICH, or is it a coincidence with ICH. All patients had risk factors for ICH like hypertension and DM. During the penetration of COVID-19 into the cells of the body, angiotensin-converting enzyme 2 (ACE2) plays a crucial role (Weimar and Kleine-Borgmann, 2017). Through ACE2, not only does the viral infection penetrate into the cell, but with COVID19 , the expression of ACE2 decreases, which leads to dysfunction of the renin-angiotensin-aldosterone system (RAAS) and damage to the lungs and other organs and systems (Weimar and KleineBorgmann, 2017). A decrease in ACE2 expression can increase the risk of ICH in several ways: (1) a decrease in ACE2 expression can increase local Angiotensin II (Ang II) levels, which, acting on AT1 receptors, can increase blood pressure; (2) ACE2 deficiency in the CNS can lead to endothelial dysfunction in the cerebral vessels, leading to an increase in the risk of a cerebral hemorrhage; (3) A decrease in ACE2 expression will also lead to a decrease in Ang (1-7) generation and depression of Ang (1-7)/MasR signaling, thereby preventing its vasodilatory, neuroprotective, and antifibrotic effects (Weimar and Kleine-Borgmann, 2017; Benger et al., 2020; South et al., 2020). Therefore, it is reasonable to conclude that COVID-19 may exacerbate hypertension and increase the risk of $\mathrm{ICH}$ in patients.

Diabetes mellitus is also an independent risk factor for the development of ICH. Several biological mechanisms could explain the observed association between high glucose and ICH. High glucose could impair normal endothelial function, and subsequently lead to brain small vessel disease (SVD). 
Degenerative changes in the walls of brain small vessels could cause ICH (Boulanger et al., 2016). Moreover, the rate of ICH in DM patients with the hypertension is higher than those without hypertension (Benger et al., 2020). Both hyperglycemia and hypertension can induce the risk factors to act on the brain vessels and to make them easy to be ruptured. DM is a chronic inflammatory condition characterized by multiple metabolic and vascular disorders that can influence our response to infection pathogens, in particular COVID-19 (Boulanger et al., 2016). Hyperglycemia and insulin resistance increase the synthesis of proinflammatory cytokines, oxidative stress, and stimulate the production of adhesion molecules that mediate tissue inflammation (Tadic et al., 2020). This inflammatory process may be one of the main mechanisms that lead to a higher propensity for COVID-19, with worse consequences in DM patients (Tadic et al., 2020). Indeed, the available data indicate that DM ranks second after hypertension among confirmed COVID-19 cases with major chronic diseases (Tadic et al., 2020).

Additionally, all of our patients had elevated D-dimer and C-reactive protein (CRP) on admission in the setting of COVID-19. The most common pattern of coagulopathy observed in patients hospitalized with COVID-19 infection is characterized by elevations in fibrinogen and D-dimer levels (Becker, 2020). In fact, patients with severe COVID-19 were reported to have increased $\mathrm{D}$-dimer and tissue plasminogen activator (tPA) plasma levels, both of which are associated with an increased propensity for hemorrhagic complications (Becker, 2020; Divani et al., 2020). Therefore, it is possible that a coagulopathy in the setting of activation of intrinsic and extrinsic fibrinolytic processes predisposed these patients to ICH. The serum inflammatory factors, such as CRP play a great role in the process of the vascular damage (Boulanger et al., 2016). Early stage COVID-19 CRP levels are known to positively correlate with lung involvement and may reflect disease severity (Wang, 2020). Some researchers found that the incidence of ICH was significantly higher in the high CRP group than in the low CRP group (Liu et al., 2014). It indicates that CRP may influence the incidence of ICH in COVID-19 patients.

\section{CONCLUSION}

While it remains to be confirmed whether there is a causal relationship between COVID-19 infection and $\mathrm{ICH}$, these three cases, along with others in the literature, support that COVID-19 patients with severe illness are at risk for ICH.

\section{REFERENCES}

Al-Dalahmah, O., Thakur, K. T., Nordvig, A. S., Prust, M. L., Roth, W., Lignelli, A., et al. (2020). Neuronophagia and microglial nodules in a SARS-CoV-2 patient with cerebellar hemorrhage. Acta Neuropathol. Commun. 8:147. doi: 10.1186/s40478-020-01024-2

Al-olama, M., Rashid, A., and Garozzo, D. (2020). COVID-19-associated meningoencephalitis complicated with intracranial hemorrhage: a case report. Acta Neurochir. (Wien) 162, 1495-1499. doi: 10.1007/s00701-02004402-w

\section{DATA AVAILABILITY STATEMENT}

All datasets presented in this study are included in the article/supplementary material.

\section{ETHICS STATEMENT}

The studies involving human participants were reviewed and approved by the Ethics Committee of the Hospital COVID19 Clinics BSMU in Ufa, Russia (within the Department of Neurosurgery), Clinic of the Bashkir State Medical University, Ufa, Republic of Bashkortostan, Russia, and Bashkir State Medical University. The patients/participants provided their written informed consent to participate in this study. Written informed consent was obtained from the individual(s) for the publication of any potentially identifiable images or data included in this article.

\section{AUTHOR CONTRIBUTIONS}

VP contributed to conception and organization of research project, drafted the main manuscript text. OB and IG performed the experiments and acquired the data. LT and AS were involved in study conception, participated in design and coordination, and helped to draft the manuscript. GA was responsible for data acquisition, writing - review and editing, analysis and interpretation of data, wrote the manuscript, and approved the final version of the manuscript. All authors have read and agreed to the published version of the manuscript.

\section{FUNDING}

This work supported by Russian Academic Excellence project "5-100" for the Sechenov University, Moscow, Russia, GALLY International Biomedical Research Institute, San Antonio, TX, United States, and Human Photosynthesis Study Center, Aguascalientes, México.

\section{ACKNOWLEDGMENTS}

We thank all participants of the underlying studies.

Bao, Y, Lin, S. Y., Cheng, Z. H., Xia, J., Sun, Y. P., Zhao, Q., et al. (2020). Clinical features of COVID-19 in a young man with massive cerebral hemorrhage-case report. SN Compr Clin Med. 2, 703-709. doi: 10.1007/s42399-020-00315-y

Becker, R. C. (2020). COVID-19 update: covid-19-associated coagulopathy. J. Thromb Thrombolysis 50, 54-67. doi: 10.1007/s11239-020-02134-3

Benger, M., Williams, O., Siddiqui, J., and Sztriha, L. (2020). Intracerebral haemorrhage and COVID-19: clinical characteristics from a case series. Brain Behav. Immun. 88, 940-944. doi: 10.1016/j.bbi.2020.06.005

Boulanger, M., Poon, M. T., Wild, S. H., and Al-Shahi Salman, R. (2016). Association between diabetes mellitus and the occurrence and outcome 
of intracerebral hemorrhage. Neurology 87, 870-878. doi: 10.1212/WNL. 0000000000003031

Daci, R., Kennelly, M., Ferris, A., Azeem, M. U., Johnson, M. D., Hamzei-Sichani, F., et al. (2020). Bilateral basal ganglia hemorrhage in a patient with confirmed COVID-19. AJNR Am. J. Neuroradiol. 41, 1797-1799. doi: 10.3174/ajnr.A6712

Divani, A. A., Andalib, S., Di Napoli, M., Lattanzi, S., Hussain, M. S., Biller, J., et al. (2020). Coronavirus disease 2019 and stroke: clinical manifestations and pathophysiological insights. J Stroke Cerebrovasc. Dis. 29:104941. doi: 10.1016/ j.jstrokecerebrovasdis.2020.104941

Dogra, S., Jain, R., Cao, M., Bilaloglu, S., Zagzag, D., Hochman, S., et al. (2020). Hemorrhagic stroke and anticoagulation in COVID-19. J. Stroke Cerebrovasc. Dis. 29:104984. doi: 10.1016/j.jstrokecerebrovasdis.2020.104984

Gogia, B., Fang, X., and Rai, P. (2020). Intracranial hemorrhage in a patient with COVID-19: possible explanations and considerations. Cureus 12:e10159. doi: 10.7759/cureus.10159

Ghani, M. U., Kumar, M., Ghani, U., Sonia, F., and Abbas, S. A. (2020). Intracranial hemorrhage complicating anticoagulant prophylactic therapy in three hospitalized COVID-19 patients. J. Neurovirol. 26, 602-604. doi: 10.1007/ s13365-020-00869-6

Haddadi, K. G., and Shafizad, M. (2020). Basal ganglia involvement and altered mental status: a unique neurological manifestation of coronavirus disease 2019. Cureus 12:e7869.

Hernández-Fernández, F., Valencia, H. S., Barbella-Aponte, R. A., ColladoJiménez, R., Ayo-Martín, Ó, Barrena, C., et al. (2020). Cerebrovascular disease in patients with COVID-19: neuroimaging, histological and clinical description. Brain awaa239. doi: 10.1093/brain/awaa239

Kannan, S., Shaik Syed Ali, P., Sheeza, A., and Hemalatha, K. (2020). COVID-19 (Novel Coronavirus 2019)-recent trends. Eur. Rev. Med. Pharmacol. Sci. 24, 2006-2011. doi: 10.26355/eurrev_202002_20378

Khattar, N. K., Sharma, M., McCallum, A. P., Oxford, B. G., Zeb, H., Suliman, S. A., et al. (2020). Intracranial hemorrhage in a young COVID-19 patient. Interdiscip. Neurosurg. 22:100878. doi: 10.1016/j.inat.2020.100878

Kim, C., Kwak, Y., Hwang, J., and Eun, M. Y. (2020). Spontaneous intracerebral hemorrhage in a patient with asymptomatic 2019 novel coronavirus disease. J. Clin. Neurol. 16, 515-517. doi: 10.3988/jcn.2020.16.3.515

Kvernland, A., Kumar, A., Yaghi, S., Raz, E., Frontera, J., Lewis, A., et al. (2020). Anticoagulation use and hemorrhagic stroke in SARS-CoV-2 patients treated at a New York healthcare system. Neurocrit. Care 1-12. doi: 10.1007/s12028-02001077-0

Li, Y., Li, M., Wang, M., Zhou, Y., Chang, J., Xian, Y., et al. (2020). Acute cerebrovascular disease following COVID-19: a single center, retrospective, observational study. Stroke Vasc. Neurol. 5, 279-284. doi: 10.1136/svn-2020000431

Liu, Y., Wang, J., Zhang, L., Wang, C., Wu, J., Zhou, Y., et al. (2014). Relationship between C-reactive protein and stroke: a large prospective community based study. PLoS One 9:e107017. doi: 10.1371/journal.pone.0107017
Morassi, M., Bagatto, D., Cobelli, M., D’Agostini, S., Gigli, G. L., Bnà, C., et al. (2020). Stroke in patients with SARS-CoV-2 infection: case series. J. Neurol. 267, 2185-2192. doi: 10.1007/s00415-020-09885-2

Nawabi, J., Morotti, A., Wildgruber, M., Boulouis, G., Kraehling, H., Schlunk, F., et al. (2020). Clinical and imaging characteristics in patients with SARSCoV-2 infection and acute intracranial hemorrhage. J. Clin. Med. 9:2543. doi: $10.3390 /$ jcm 9082543

Poyiadji, N., Shahin, G., Noujaim, D., Stone, M., Patel, S., and Griffith, B. (2020). COVID-19-associated acute hemorrhagic necrotizing encephalopathy: CT and MRI features. Radiology 296, E119-E120. doi: 10.1148/radiol.202020 1187

Reddy, S. T., Garg, T., Shah, C., Nascimento, F. A., Imran, R., Kan, P., et al. (2020). Cerebrovascular disease in patients with COVID-19: a review of the literature and case series. Case Rep. Neurol. 12, 199-209. doi: 10.1159/00050 8958

Shahjouei, S., Naderi, S., Li, J., Khan, A., Chaudhary, D., Farahmand, G., et al. (2020). Risk of stroke in hospitalized SARS-CoV-2 infected patients: a multinational study. EBioMedicine 59:102939. doi: 10.1016/j.ebiom.2020. 102939

Sharifi-Razavi, A., Karimi, N., and Rouhani, N. (2020). COVID-19 and intracerebral haemorrhage: causative or coincidental? New Microbes New Infect. 35:100669. doi: 10.1016/j.nmni.2020.100669

South, A. M., Diz, D. I., and Chappell, M. C. (2020). COVID-19, ACE2, and the cardiovascular consequences. Am. J. Physiol. Heart Circ. Physiol. 318, H1084-H1090. doi: 10.1152/ajpheart.00217.2020

Tadic, M., Cuspidi, C., and Sala, C. (2020). COVID-19 and diabetes: is there enough evidence? J. Clin. Hypertens. (Greenwich) 22, 943-948. doi: 10.1111/jch. 13912

Wang, L. (2020). C-reactive protein levels in the early stage of COVID19. Med. Mal. Infect. 50, 332-334. doi: 10.1016/j.medmal.2020. 03.007

Weimar, C., and Kleine-Borgmann, J. (2017). Epidemiology, prognosis and prevention of non-traumatic intracerebral hemorrhage. Curr. Pharm. Des. 23, 2193-2196. doi: 10.2174/138161282266616102715 2234

Conflict of Interest: GA employed by GALLY International Biomedical Research LLC. All of other authors declared no conflict of interest.

Copyright (c) 2020 Pavlov, Beylerli, Gareev, Torres Solis, Solís Herrera and Aliev. This is an open-access article distributed under the terms of the Creative Commons Attribution License (CC BY). The use, distribution or reproduction in other forums is permitted, provided the original author(s) and the copyright owner(s) are credited and that the original publication in this journal is cited, in accordance with accepted academic practice. No use, distribution or reproduction is permitted which does not comply with these terms. 